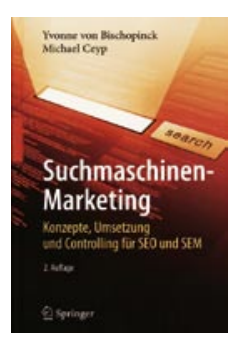

Suchmaschinen-Marketing hat sich heutzutage als fester Bestandteil des Online-Marketing im Markt etabliert und kaum ein Unternehmen kann es sich eigentlich leisten, dieses Instrument nicht als integralen Bestandteil des Kommunikations-Mix einzusetzen. Schließlich ist es das Ziel des Suchmaschinen-Marketing, die Sichtbarkeit der Produkt-, Marken- oder Unternehmensinformationen innerhalb der Ergebnisleisten der Suchmaschinen zu verbessern und somit interessierte Personen über organische oder bezahlte Links auf die eigenen Webseiten zu lenken. Doch in vielen Unternehmen fehlt immer noch das Wissen zum systematischen Einsatz dieses Online-Marketinginstruments, obwohl die Relevanz des Internets als Informationsmedium aufseiten der Konsumenten stetig an Bedeutung gewinnt.

Von Bischopinck und Ceyp nehmen sich dieser Problemstellung an und präsentieren mit ihrem Buch einen äußerst umfassenden und anwendungsorientierten Einblick in die Grundlagen des Suchmaschinen-Marketing. Das Buch gliedert sich dabei in zwei zentrale Abschnitte. Während der erste Part eine theoretisch aufgearbeitete Hinführung zu den Konzepten, zur Umsetzung und zum Controlling darstellt, zeigt der zweite Teil äußerst praxisnah die operative Implementierung des Suchmaschinen-Marketing anhand von zwei ausgewählten Fallstudien auf. Die Relevanz, aber auch die Dynamik dieses Themas zeigen sich insbesondere in der Tatsache, dass dieses Buch innerhalb kurzer Zeit bereits in der zweiten, überarbeiteten, aktualisierten und erweiterten Auflage erscheint. Gegenüber der vorhergehenden Ausgabe wurde insbesondere der Inhalt dem neuesten Stand der Forschung angepasst und an manchen Stellen deutlich vertieft. Zudem wurde eine weitere Case Study aufgenommen, welche den Unternehmen eine zusätzliche Hilfestellung bei der Integration des Suchmaschinen-Marketing in den eigenen Kommunikations-Mix bietet.

Die Autoren bieten mit ihrem Werk einen praxisorientierten Leitfaden für Unternehmen, die sich mit den Fragestellungen und Möglichkeiten des Suchmaschinen-Marke- ting vertieft auseinandersetzen möchten. Es gelingt von Bischopinck und Ceyp, den interessierten Lesern ein aktualisiertes und erweitertes Kompendium an die Hand zu geben, das die Fragen zum Suchmaschinen-Marketing auf verständliche Weise und vollumfänglich beantwortet.

V. Walter

\section{Der Transfer der Marktorientierung über Hierarchieebenen}

Kraus, Florian: Der Transfer der Marktorientierung über Hierarchieebenen: Eine empirische Mehrebenenuntersuchung, Wiesbaden: Gabler, 363 Seiten, EUR (D) 59,90, ISBN 978-3-8349-1321-0

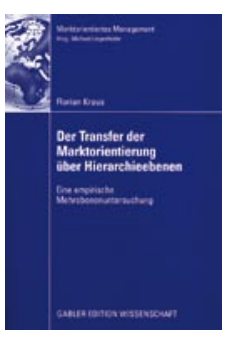

Die Dissertationsschrift von Dr. Florian Kraus beschäftigt sich auf Basis einer empirischen Untersuchung, die gemeinsam mit einer der größten Reisevertriebsorganisationen Deutschlands durchgeführt wurde, mit dem Transfer der Marktorientierung über verschiedene Hierarchiestufen. Aus der Bedeutung der Marktorientierung als zentrales Marketingkonstrukt zur Beeinflussung des Unternehmenserfolgs erschließt sich die hohe Relevanz der Arbeit. Wenig bekannt ist bisher allerdings noch, wie sich die Marktorientierung innerhalb von einem Unternehmen überträgt und welchen Einfluss dieser Transfer auf Kunden und letztendlich den Unternehmenserfolg hat.

Auf der Basis ausgewählter theoretischer Konzepte entwickelt Florian Kraus in diesem Buch ein vier Forschungsebenen umfassendes Untersuchungsmodell zur Übertragung der Marktorientierung auf verschiedene Hierarchieebenen und den Einfluss auf die Kunden. Mithilfe einer großangelegten Mehrebenenanalyse nimmt er eine empirische Überprüfung seines Modells am Beispiel eines realen Vertriebssystems vor. Die Datengrundlage hierfür bilden umfangreiche Befragungen von Top-Managern, Verkaufsmanagern, Kundenkontaktmitarbeitern und Kunden. Ebenso werden unterschiedliche Wirkungszusammenhänge auf Basis der internen und externen Unternehmenssituation identifiziert und berücksichtigt. Die ge- wonnenen Erkenntnisse münden in der Ableitung konkreter Handlungsempfehlungen für die Unternehmenspraxis auf den Ebenen Top-Manager, Verkaufsmanager und Kundenkontaktmitarbeiter sowie in Anknüpfungspunkten für die weitere wissenschaftliche Forschung.

Dem Autor gelingt es, die aufgeworfenen Fragen durch seine Arbeit nachvollziehbar und hinreichend zu beantworten. Insbesondere die umfangreiche quantitative Untersuchung liefert viele Erkenntnisse darüber, wie genau der Transfer der Markorientierung über Hierarchieebenen vonstattengeht, wie die zugrundeliegenden Mechanismen beeinflusst werden und welche Erfolgswirkung Unternehmen damit erzielen können. Neben der umfassenden Literaturbestandsaufnahme werden auch die theoretischen und methodischen Grundlagen der Untersuchung sehr sorgfältig und gut nachvollziehbar dargestellt. Diese Arbeit richtet sich daher ebenso an Führungskräfte in Dienstleistungsunternehmen in den Bereichen Vertriebs- und Personalmanagement als auch an Dozenten und Studenten der Betriebswirtschaftslehre mit den Schwerpunkten Marketing und Handelsbetriebslehre. Durch ihren hohen Neuigkeitsgehalt ist diese Arbeit für beide Zielgruppen gleichermaßen empfehlenswert.

D. Herhausen 